\title{
Acute granulomatous interstitial nephritis and ulcerative colitis: a case report and literature review
}

\author{
Giorgia Comai, ${ }^{1}$ Olga Baraldi, ${ }^{1}$ Vania Cuna, ${ }^{1}$ Valeria Corradetti, ${ }^{1}$ Maria Cappuccilli, ${ }^{1}$ Marco Busutti, ${ }^{1}$ \\ Deborah Malvi, ${ }^{2}$ Benedetta Fabbrizio, ${ }^{2}$ Massimo Campieri, ${ }^{3}$ Gaetano La Manna ${ }^{1}$ \\ ${ }^{1}$ Department of Experimental Diagnostic and Specialty Medicine, Nephrology, Dialysis and Renal Transplant Unit; ${ }^{2}$ Pathology \\ Department; ${ }^{3}$ Department of Clinical Medicine, S. Orsola-Malpighi Hospital, University of Bologna, Bologna, Italy
}

\begin{abstract}
Tubulo-interstitial nephritis (TIN) in patients affected by inflammatory bowel disease, both ulcerative colitis and Crohn's disease, is usually considered as drug-associated to aminosalicylate. We report a rare case of granulomatous active tubulo-interstitial nephritis in a young patient with a recent diagnosis of ulcerative colitis naïve to aminosalicylate treatment. The patient has been successfully treated with steroids administration. Our purpose is to sensitize that TIN should always to be considered in differential diagnosis an extra-intestinal manifestation of bowel disease.
\end{abstract}

\section{Introduction}

Extra-intestinal manifestations are rather common in inflammatory bowel disease (both Crohn's disease

Correspondence: Gaetano La Manna, Department of Experimental, Diagnostic and Specialty Medicine (DIMES), Nephrology, Dialysis and Transplantation Unit, St. Orsola Hospital, University of Bologna, via G. Massarenti 9 (Pad. 15), 40138 Bologna, Italy.

Tel.: +39.051.2144577 - Fax: +39.051.344439

E-mail: gaetano.lamanna@unibo.it

Key words: Aminosalicylate therapy; inflammatory bowel disease; tubulo-interstitial nephritis; steroid therapy.

Contributions: GC, study design, clinical management, data collection and interpretation, manuscript preparation, literature search; $\mathrm{OB}$, clinical management, data collection and interpretation, literature search; VC1, data collection, clinical management, literature search; VC2, study design, data interpretation, manuscript preparation, literature search; $\mathrm{MC} 1$, manuscript preparation, literature search; $\mathrm{MB}$, clinical management, data collection; DM, processing and interpretation of histological samples; BF, processing and interpretation of histological samples; MC2, main coinvestigator, study design; GLM, main investigator: study design, coordination, supervision.

Conflict of interest: the authors declare no potential conflict of interest.

Received for publication: 16 September 2017.

Accepted for publication: 16 October 2017.

This work is licensed under a Creative Commons Attribution NonCommercial 4.0 License (CC BY-NC 4.0).

CCopyright G. Comai et al., 2018

Licensee PAGEPress, Italy

Italian Journal of Medicine 2018; 12:57-60

doi:10.4081/itjm.2018.929 and ulcerative colitis) with a reported prevalence that ranges from $6 \%$ to $46 \%$ of patients. ${ }^{1}$ Renal and urological involvement has a prevalence of $4-23 \%$ and is mainly represented by nephrolithiasis, urinary fistulas and malignancies in particular renal cell carcinoma commonly diagnosed at a younger age compared to the general population..$^{2-4}$ Parenchymal renal involvement is a rare but serious extra-intestinal manifestation that can affect both glomerular and tubulo-interstitial compartment. ${ }^{5}$ The pathogenesis of kidney damage in inflammatory bowel disease (IBD), as in other extraintestinal manifestation, has not been completely understood; the main hypotheses consider that it is related to intestinal disease activity and dependent on the same immunological mechanism or, alternatively, as a result of systemic autoimmune deregulation. ${ }^{6}$ Moreover drug toxicity can be considered as a relevant cause of kidney damage in IBD patients. ${ }^{7}$ The most common glomerulonephritis are immunoglobulin (Ig)A nephropathy, membranous glomerulonephritis, focal segmental glomerulosclerosis and amyloidosis, whereas the most common types of tubulointerstitial nephritis are drug-related or not drug-related. ${ }^{3}$

\section{Case Report}

A 37-year-old man with a past history of severe obesity [body mass index (BMI) $>35$ ] and mild hypertension, successfully treated with $\beta$-blocker, a family history positive for ulcerous colitis (father) and kidney disease (cousin with end stage kidney disease for unknown nephropathy), with a recent histological diagnosis of active ulcerative colitis established after 4 months of changing bowel habits, abdominal pain and mild fever.

Patient had been referred to nephrology clinic by 
a gastroenterologist after the finding of progressive worsening in renal function (serum creatinine baseline 1 year earlier was $0.8 \mathrm{mg} / \mathrm{dL}, 6$ months after 1.6 $\mathrm{mg} / \mathrm{dL}$ increased to $2.2 \mathrm{mg} / \mathrm{dL}$ at the moment of the evaluation, estimated glomerular filtration rate (eGFR) CKD-EPI $47 \mathrm{~mL} / \mathrm{min} / 1.73 \mathrm{~m}^{2}$ ) associated with microhematuria and sub-nephrotic proteinuria ( $0.4 \mathrm{gr} /$ day). No 5-acetylsalycilic acid (ASA) was administered in order to avoid confounding factors on renal picture. Azathioprine (50 mg twice daily) was prescribed to control intestinal symptoms.

Physical examination at the admission revealed a blood pressure of $110 / 60 \mathrm{mmHg}$, heart rate of $76 \mathrm{bpm}$, weight of $93 \mathrm{~kg}\left(\right.$ BMI $\left.29 \mathrm{~kg} / \mathrm{m}^{2}\right)$. Blood tests shown a serum creatinine of $2.27 \mathrm{mg} / \mathrm{dL}$, eGFR CKD-EPI 45 $\mathrm{mL} / \mathrm{min} / 1.73 \mathrm{~m}^{2}$, anemia (hemoglobin $12 \mathrm{~g} / \mathrm{dL}$ ), white blood cells count count and C-reactive protein were normal, serum electrolytes were in optimal range, parathyroid hormone $51 \mathrm{pg} / \mathrm{mL}$. Urinary abnormalities have been confirmed, urine culture was negative.

Immunological tests such as antinuclear antibodies, anti-neutrophil cytoplasmic antibodies, anti-double stranded DNA antibodies, C3/C4 fragment of complement system, and rheumatoid factor were normal.

Abdomen ultrasound showed kidneys of normal size and echogenicity, without structural abnormalities. A kidney biopsy was performed.

In kidney biopsy, light microscopy demonstrated intense and diffuse interstitial infiltration by inflammatory cells including lymphocytes and monocytes, leukocyte infiltration of the tubular epithelium called tubulitis and a small amount of tubular necrosis. There were also a lot of interstitial granulomata with epithelioid histiocytes and a few giant cells without caseous necrosis. The glomeruli were normal. Immunofluorescence was negative for all serum tested, a nonspecific granular positivity for $\mathrm{C} 3$ was present in some tubular basement membranes. Electron microscopic showed the presence of interstitial inflammatory infiltration of lympho-monocytes also present in peritubular capillaries. The diagnosis was acute granulomatous interstitial nephritis (Figure 1).

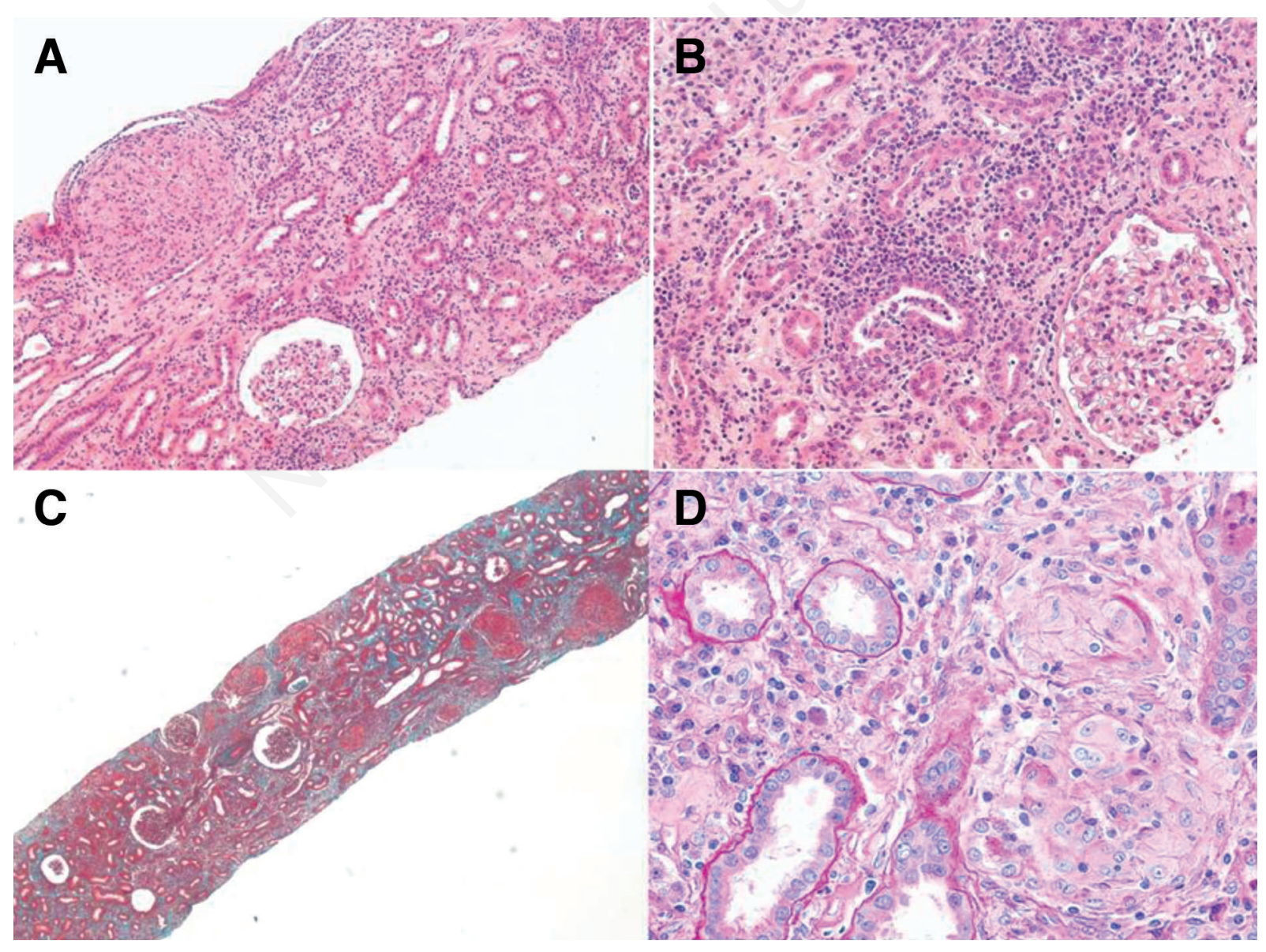

Figure 1. Light microscopic of the kidney biopsy showing interstitial infiltration by inflammatory cells, a huge number of granulomata with epithelioid histiocytes and a few giant cells without caseous necrosis. A) Hematoxylin and eosin (H\&E) 10×; B) H\&E 20×; C) Masson Trichrome 4×; D) PAS 40×. 
The diagnosis of extra-intestinal manifestation of ulcerative colitis has been placed once other causes of granulomatous interstitial nephritis were excluded: quantiferon test for tuberculosis was negative; angiotensin converting enzyme serum levels, high-resolution computed tomography of the lung and whole body Fluorine-18 fluorodeoxyglucose positron emission tomography were performed in order to exclude sarcoidosis.

Patient has been treated with high-dose intravenous methylprednisolone bolus $(250 \mathrm{mg} \times 3)$ gradually tapered to oral $32 \mathrm{mg}$ of prednisone daily, associated with azathioprine for the ulcerative colitis treatment (50 mg/daily).

Blood tests performed after 1 month of therapy shown significant improvement in renal function, serum creatinine was $1.69 \mathrm{mg} / \mathrm{dL}$ (eGFR CKD-EPI 62 $\mathrm{mL} / \mathrm{min} / 1.73 \mathrm{~m}^{2}$ ) and complete regression of intestinal symptoms; blood pressure is well controlled without anti-hypertensive drugs.

\section{Discussion and Conclusions}

Acute granulomatous interstitial nephritis is considered an uncommon pathological finding in renal biopsy ( $<1 \%$ of native renal biopsies) that has been associated with various etiologies, of which drug hypersensitivities and sarcoidosis encompass the majority of cases. ${ }^{8}$

Ulcerative colitis is a chronic inflammatory disease with localization in the colon and rectum. The exact pathogenesis is not fully clarified but involves a dysregulation of the gastrointestinal immune system in patients with a genetic predisposition. Extraintestinal manifestations are common (up to $40 \%$ of patients) and they are associated with autoimmune mechanism. ${ }^{9}$

In our case we observed a progressive worsening of renal function in a patient with a recent finding of ulcerative colitis without an ongoing treatment with mesalazine or other nephrotoxic drugs. Since our patient had not used any drugs, we could postulate that the main pathogenetic mechanism of renal injury was the disease activity of ulcerative colitis.

Parenchymal renal disease in IBD has been described but granulomatous interstitial nephritis is considered extremely rare.

In current literature many case reports describe renal involvement in patients affected by ulcerative colitis but systematic epidemiological studies are lacking. Ambruzs et al. published in 2013 the most comprehensive case series on histopathological findings in IBD patients: they retrospectively review all native kidney biopsy examined and of 33,713 patients, 83 where affected by inflammatory bowel disease; the most frequent diagnosis was IgA nephropathy (24\%), followed by interstitial nephritis (19\%), nephrosclero- sis $(12 \%)$, acute tubular injury $(8 \%)$ and proliferative glomerulonephritis (7\%) equally distributed between Crohn's disease and ulcerative colitis. The interstitial nephritis cases where classified as acute, granulomatous and chronic; all granulomatous cases where related to exposure to 5-ASA. ${ }^{10}$

In literature most cases of tubulo-interstitial nephritis (TIN) are linked to 5-aminosalycilate exposure, nevertheless is not always possible to discern if kidney damage originated from extra-renal manifestations of bowel disease or drug-induced toxicity. ${ }^{7}$ Lately some case reports described patients with TIN not related to drugs exposure ${ }^{11}$ or as primary manifestation of bowel disease. ${ }^{10}$ Moreover, recent studies showed that tubular damage is tensely related to disease activity rather than drugs and that TIN biopsyproven in patients with IBD and absent 5-ASA exposure, progressed independently of the intestinal disease activity. ${ }^{12,13}$

Recent promising studies have reported as the expression of some protein biomarkers, such as neutrophil gelatinase-associated lipocalin, a molecule with multiple activities, including anti-inflammatory and immunomodulatory effects, can provide information able to early detect IBD activity such as possible renal damage. ${ }^{14,15}$

In conclusion, granulomatous interstitial nephritis is a rare extraintestinal manifestation of ulcerative colitis, which is potentially due to systemic immune dysregulation and T-cell activation that it could be treated successfully with immunosuppression. We suggest that kidney function should be monitored and a renal biopsy should be considered for those patients with a persistent increase in serum creatinine.

\section{References}

1. Mendoza JL, Lana R, Taxonera C, et al. Extraintestinal manifestations in in ammatory bowel disease: differences between Crohn's disease and ulcerative colitis. Med Clin (Barc) 2005; 125:297-300.

2. Pardi DS, Tremaine WJ, Sandborn WJ, McCarthy JT. Renal and urologic complications of inflammatory bowel disease. Am J Gastroenterol 1998;93:504-14.

3. Corica D, Romano C. Renal involvement in inflammatory bowel diseases. J Crohn Colitis 2016:226-35.

4. Brunocilla E, Borghesi M, Schiavina R, et al. Small renal masses initially managed using active surveillance: results from a retrospective study with long-term follow-up. Clin Genitourin Cancer 2014;12:178-81.

5. Oikonomou K, Kapsoritakis A, Eleftheriadis T, et al. Renal manifestations and complications of in ammatory bowel disease. In Amm Bowel Dis 2011;17:1034-45.

6. Danese S, Semeraro S, Papa A, et al. Extraintestinal manifestations in inflammatory bowel disease. World $\mathrm{J}$ Gastroenterol 2005;11:7227-36.

7. Gisbert JP, Gonzàlez-Lama Y, Maté J. 5-Aminosalicylates and renal function in inflammatory bowel disease: 
a systematic review. In Amm Bowel Dis 2007;13: 629-38.

8. Timmermans S, Christiaans M, Abdul-Hamid M, et al. Granulomatous interstitial nephritis and Crohn disease. Clin Kidney J 2016;9:556-9.

9. Tokuyama H, Wakino S, Konishi K, et al. Acute interstitial nephritis associated with ulcerative colitis. Clin Exp Nephrol 2010;14:483-6.

10. Ambruzs JM, Walker PD, Larsen CP. The histopathologic spectrum of kidney biopsies in patients with inflammatory bowel disease. Clin J Am Soc Nephrol 2014;9:265-70.

11. Izzedine H, Simon J, Piette AM, et al. Primary chronic interstitial nephritis in Crohn's disease. Gastroenterology 2002;123:1436-40.

12. Poulou AC, Goumas KE, Dandakis DC, et al. Micropro- teinuria in patients with inflammatory bowel disease: is it associated with the disease activity or the treatment with 5-aminosalicylic acid? World J Gastroenterol 2006;12:739-46.

13. Fraser JS, Muller AF, Smith DJ, et al. Renal tubular injury is present in acute inflammatory bowel disease prior to the introduction of drug therapy. Aliment Pharmacol Ther 2001;15:1131-7.

14. La Manna G, Ghinatti G, Tazzari PL, et al. Neutrophil gelatinase-associated lipocalin increases HLA-G+/Fox P3+ T-regulatory cell population in an in vitro model of PBMC. PLoS One 2014;9:1-9.

15. Thorsvik S, Damås JK, Granlund AV, et al. Fecal neutrophil gelatinase-associated lipocalin as a biomarker for inflammatory bowel disease. J Gastroenterol Hepatol 2017;32:128-35. 